\title{
ABDOMINAL PERIAORTITIS IN A MALE SYSTEMIC LUPUS ERYTHEMATOSUS PATIENT
}

Thaíssa Antunes Mattar Valente ${ }^{1,{ }^{*}}$, Natalia Pereira machado1, Fábio Henrique Carneiro ${ }^{1}$, Ana Cristina Boni Brandão Lenci Marques $^{1}$, Marina Machado pereira ${ }^{1}$, André Wilson de Lima Oliveira ${ }^{1}$, Thais Miranda da Silva de Andrade ${ }^{1}$, Roberta Zawadzki Bueno ${ }^{1}$, Gabriel Caetano Pereira ${ }^{1}$, Elisa Cristina Pêgas ${ }^{1}$, Aline Hikari Ishida ${ }^{1}$

1.Universidade Federal do Paraná, Curitiba (PR), Brazil.

*Corresponding author: thaissavalente@hotmail.com

\section{BACKGROUND}

Chronic periaortitis is a rare entity characterized by a fibroinflammatory tissue surrounding most commonly abdominal aorta and iliac arteries, which can spread to retroperitoneum, and is of unknown etiology. Other vascular districts which may be involvedalbeit rarely - are the coronary, renal, mesenteric and celiac arteries. Firstly, it was thought to be caused by a local inflammatory reaction to antigens in the atherosclerotic plaques in the aorta, but now it has been considered a primary inflammatory or immune mediated disorder because of its association with immunoregulatory diseases such as systemic lupus erythematosus (SLE), Sjögren's syndrome and systemic vasculitis.

\section{CASE REPORT}

A 20-year-old Brazilian brown-skinned patient of male sex was admitted to a hospital with moderate abdominal pain, nausea, constipation and inability to have a bowel movement or pass gas in the last 3 days. He had a history of SLE and antiphospholipid syndrome (APS), diagnosed in 2018. He was in use of warfarin, prednisone $5 \mathrm{mg} /$ day, hydroxychloroquine $400 \mathrm{mg} / \mathrm{day}$ and mycophenolate mofetil $2 \mathrm{~g} /$ day. He also had bicuspid aortic valve and moderate regurgitation with no cardiac insufficiency. His initial laboratory findings showed: 9420 white blood cell count/mm3; hemoglobin 16.3 g/dL; platelet 194,000/mm3; erythrocyte sedimentation rate $1 \mathrm{~mm} / \mathrm{h}$, and C-reactive protein (CRP) $3.38 \mathrm{mg} / \mathrm{dL}$ (normal 0.02-0.50 mg/dL). He had no clinical or laboratory signs of infection or other lupus flare. An abdominal computed tomography (CT) scan revealed diffuse infiltrative soft tissue attenuation around the celiac trunk and superior mesenteric artery near their origin from abdominal aorta. Because of technical difficulties in assessing the lesion histopathologically, and the lack of a massive (neoplasm) appearance, presumptive diagnosis of periaortitis was made, and he was given three consecutive pulses of methylprednisolone $1 \mathrm{~g}$, his mycophenolate dose was raised to $3 \mathrm{~g} / \mathrm{day}$ and he was discharged 3 days after with prednisone $60 \mathrm{mg} /$ day and resolution of abdominal symptoms. Two months later, a new abdominal CT was taken with complete resolution of the findings and normalization of CRP.

\section{CONCLUSION}

We describe a case of a man without major atherosclerosis, with diagnosis of SLE and APS, presenting with periaortitis in the celiac trunk, rare manifestation and location of periaortitis, due to a lupus flare, which showed rapid improvement after initiation of immunosuppressive therapy. This case reassures the importance of considering this serious cause of abdominal pain in a SLE and or APS patient in the differential diagnosis.

\section{KEYWORDS}

Periaortitis, Systemic lupus erythematosus, Antiphospholipid syndrome. 